\title{
THE ALGOLS, RED SPECTRA, BE STARS, AND EVEN NEUTRINOS
}

\author{
M. PLAVEC and R. S. POLIDAN \\ Dept. of Astronomy, University of California, Los Angeles, Calif., U.S.A.
}

\begin{abstract}
The introduction of image tubes makes spectroscopic studies in the red and near infrared spectral regions much more efficient. Work currently done at the Lick Observatory by the Los Angeles binary star investigators is reported. Preliminary results on element abundances in the subgiant in U Sagittae indicate that there may indeed exist systematic abundance anomalies of potential relevance to the problem of the solar neutrino flux. Emission at $\mathrm{H} \alpha$ has been observed in many systems, and several types of circumstellar features are indicated. It is suggested that some Be stars and shell stars are interacting binaries. The possibility of observing very deep stellar eclipses is discussed.
\end{abstract}

\section{Matching the Computed Models with Real Stars}

It was the effort to explain the evolutionary paradox of the Algols systems that led to the theory of large-scale mass transfer in close binary stars. When the problem was solved in principle, attention shifted to other areas, in particular when the binary X-ray sources were discovered. The problem of the Algols has not been completely solved. In a review written three years ago (Plavec, 1973) it was emphasized that substantial progress would require close cooperation between observers and theorists. Recent developments are very promising in this respect.

The case in point is the recent investigation of the eclipsing binary AS Eri. This Algol system has been known since 1946 (Gaposchkin, 1946) to be one of thase having extremely small mass function (0.003). However, as is usual, the secondary spectrum was not observable. More recently, Popper (1973) managed to detect and to measure the $\mathrm{D}$ lines of the secondary star, and to derive a complete set of reliable elements of the system in combination with the photometric work by Koch (1960). The primary component is an ordinary main-sequence star, $\mathrm{A} 3 \mathrm{~V}$, with a mass of $1.9 M_{\odot}$. The secondary is approximately G9 IV, with mass only $0.21 M_{\odot}$, but surpassing in visual (and bolometric) magnitude the Sun by about 1 mag. This extremely small mass, combined with considerable overluminosity, perplexed the prominent astrophysicists in the fifties (cf. Struve, 1950; Kopal, 1959), but is now perfectly well explained in terms of mass transfer of mode B. Popper remarks quite rightly:

... it appears to me that this prediction of the properties of the secondary of AS Eri is an outstanding success of the theory of mass exchange.

However, equally justified is the appreciation of the observing astronomer's work by the theoreticians. Refsdal et al. (1974) say:

New standards on the observational side were set by the recent rediscussion of AS Eri by Popper (1973) who found encouraging agreement with the available theory. The observational data derived there are so accurate that, for the first time, it seems reasonable to analyze a specific system ... For this, however, we have to improve also the rather idealized previous theory.

These two statements symbolize very nicely the new and exciting stage of the investigation of the Algol-type semi-detached binaries. The idealization of the theoretical models consisted mainly in the assumption of conservation of mass and orbital angular momentum in the system; in that the accretion processes and the behavior of the mass-gaining star were 
ignored; and in that no attention was paid to the actual processes of mass transfer. This Symposium more than anything else signals the profound change in the theoretical approach: all the problems mentioned are being vigorously attacked by brilliant theorists and no doubt the future evolutionary model sequences will be much more physically realistic than the previous ones.

However, there is little doubt that theory itself will not be able to give an unambiguous description of the evolution. Refsdal et al. (1974) show that very good knowledge of the present status of the system like AS Eri still permits a considerable latitude of solutions describing its past history. Moreover, a number of physically plausible processes affecting the system is certainly possible, and it is the observation of many binaries that must help to decide what is actually happening and what most probably happened in the past.

It is therefore very gratifying to realize that, parallel to the theory, observations have made great strides forward, too. The new driving factor is the introduction of excellent image tubes giving very good red and near-infrared spectra with a speed far surpassing everything known before. The excellent Varo image tube of the Lick Observatory, attached to the coude spectrograph of the 120-in. telescope, is one of these devices, and the binary star group at the University of California campus in Los Angeles has been using it systematically to improve our knowledge of the Algol systems (and, incidentally, of a far larger group of stars including, besides the well-established eclipsing binaries, also Be stars, shell stars, symbiotic variables, and various peculiar objects).

Coming soon is a study of BM Ori, the eclipsing binary in the Orion Trapezium (Popper and Plavec, 1974, 1976). This system was suspected of harboring a black hole (Wilson, 1972) since during the apparently total eclipse of the B3 star in the system, only the B3 spectrum was recorded by Doremus (1970). The higher dispersion of the Lick spectra and the use of the Varo tube permitted us to detect the spectrum of the secondary component and to measure its D lines. The secondary component is most likely a late A giant, still contracting to the main sequence. The peculiar character of the light curve seems to be best explained by the suggestion originally made by Hall (1971), namely that the secondary star is rotating differentially (along the radius), and is best described by the models of Bodenheimer and Ostriker (1970), which can give flattened, disk-shaped stars. This is the first observational indication that such stars do indeed exist.

Going back to the genuine Algol systems, Popper's systematic effort promises to yield soon radial velocity curves of the secondaries of RY Gem, TT Hya, AW Peg, TW Dra, and TW And, based again on their Dlines. The first three are rather long-period systems, where the secondary star is a giant and gives enough light in the D region to show its Dlines in the combined spectrum with an early A primary.

Systems like USge and U Cep are less favorable. The primary is more luminous, being a late B star; the secondary is a subgiant, the system is closer, and the secondary obviously rotates in synchronism. Since the synchronized rotation velocity is about $60 \mathrm{~km} \mathrm{~s}^{-1}$ or more, its lines are fuzzy and weaker.

Really good, in fact pure, secondary spectra of U Sge and U Cep can be obtained only during the total phase of the primary eclipse. Unfortunately, this phase is rather short: it lasts some $90 \mathrm{~min}$ for USge and $110 \mathrm{~min}$ for U Cep. This time interval is comparable to the exposure time required to obtain just one good blue spectrogram of the system at this phase at about $20 \AA \mathrm{mm}^{-1}$, even with the best available spectrograph and a large telescope. In order to get the slope of the radial velocity curve of the secondary of USge, 
Joy (1930) had to employ a dispersion of $75 \AA \mathrm{mm}^{-1}$. At this dispersion, however, it is simply impossible to obtain good radial velocities of a G3 subgiant with rotationally broadened lines. Thus the masses in USge remain uncertain within too wide limits to warrant a refined calculation of evolutionary models.

Again, the use of the Varo tube at the Lick 120-inch coude spectrograph promises great improvement. We were able to obtain several sequences of very good $17 \AA \mathrm{mm}^{-1}$ spectra in the $\mathrm{H} \alpha$ region for both USge and U Cep at primary minimum, with exposure times of 10 to $15 \mathrm{~min}$. The lines are then much less smeared by orbital motion; moreover, blending is much less severe in the $\mathrm{H} \alpha$ region. Therefore, we hope to derive much better values for the slopes of the radial velocity curves. Similar sets of secondary spectra have already been obtained also for S Equ and AW Peg, and no doubt many other systems are within our reach. The cool subgiant or giant component often dominates the combined spectrum in the red or near infrared region for a considerable part of the partial eclipse, so that even partially eclipsing systems like $\mathrm{U} \mathrm{CrB}$ are tractable. If the blending at $\mathrm{H} \alpha$ is still serious, one can go farther out in the infrared, up to the Ca II triplet at about $\lambda 8600 \AA$. The potential of the equipment at these wavelengths has not even been fully tested, although considerable material has already been accumulated on the appearance of the Ca II triplet itself.

\section{The Puzzle of the Abundances}

The availability of pure and unsmeared spectra of the secondary components will help to solve the puzzling problem of their anomalous abundances. This problem gradually emerged over the years as more and more observers reported large ultraviolet excesses $\delta(U-V)$ associated with the cool components of many systems. In single stars, metal deficiency is often invoked as the cause of such an excess. However, in binary systems the problem is much more complex, and Koch (1972) quite justly remarks that broad-band photometry may very well respond primarily to circumstellar gas rather than signal abundance anomalies.

Somewhat more weight should be attached to the findings of specially arranged narrowband photometry. Miner (1966) measured essentially the relative strength of the G band, which to a large degree reflects the combined strength of metallic lines. Systematic underabundance of metals was indicated in his study for a number of evolved binaries (U Cep, TW Dra, U Sge, X Tri, RY Per, and some members of the AR Lac/RS CVn group). The average logarithmic deviation for 12 secondaries was $[\mathrm{Fe} / \mathrm{H}]=-0.96$. The result for U Cep was checked and confirmed by McNamara (1967). Hall (1967) is another narrowband photometric study found indication that the $\mathrm{CN}$ band at $\lambda 4200 \AA$, too, is systematically weakened in the evolved secondaries, which may imply underabundance of $\mathrm{CN}$.

While such photometric studies can signal abundance deviations, nothing can replace actual detailed analysis of the spectra. Here we run into the difficulties caused by faintness of the stars, short duration of total eclipses, and advanced spectral types. Bond (1972) observed the secondary of S Vel, an unusually late-type giant (K5 III), and concluded that his observations are far better accounted for by a moderate metal deficiency than by circumstellar gas cloud. With a dispersion of only $125 \AA \mathrm{mm}^{-1}$, he, of course, could not study any details and only compared the spectrum features with $\delta$ Lep, a high-velocity red giant believed to be moderately metal-deficient. A similar 
comparison was undertaken by Baldwin (1973) for U Cep. Although his dispersion was much more favorable to reliable comparison $\left(40 \AA \mathrm{mm}^{-1}\right)$, he found no evidence of metal deficiency, contrary to the photometric results by Miner and McNamara.

The epoch of actual spectroscopic analyses started with the work of Naftilan (1975a). By use of the powerful technique of spectrum synthesis, he analyzed spectra of three Algols, namely UCep, TT Hya, and ZZ Cnc, and of two objects of the RSCVn type (RSCVn and RW UMa). The Algol subgiants may show a very mild metal underabundance, but the deviations Naftilan found are within the observational uncertainty established for standard stars, $[\mathrm{Fe} / \mathrm{H}]= \pm 0.3$. For the two RSCVn-type objects the underabundancy of metals appears to be more definitely established and close to one order of magnitude. The greatest difficulty in Naftilan's analysis was again the relatively low dispersion, namely $39 \AA \mathrm{mm}^{-1}$. With the lines as a rule substantially broadened by rotation and some other factor (microturbulence?), the blends are serious in the synthesized regions which do not go beyond $\lambda 4800 \AA$.

The more recent investigation by Naftilan (1975b) on U Sge is based on a somewhat more adequate plate material. He had at his disposal a plate taken by Popper at $16 \AA$ $\mathrm{mm}^{-1}$, and several spectrograms obtained in a joint effort by Batten, Grygar, and Plavec at Victoria in 1969/1970, with dispersions ranging up to $15 \AA^{-1} \mathrm{~mm}^{-1}$. Blending still remains a problem, and the results obtained so far must be considered as preliminary, mainly because of the narrow region actually synthesized. Nevertheless, the results are very interesting and of potentially great impact. First of all, one realizes that it is impossible to lump all heavier elements together, and to speak in general terms of metal underabundance. The following Table I, prepared by Naftilan, shows interesting groupings.

The elements of the iron peak - $\mathrm{Fe}, \mathrm{Ti}, \mathrm{Cr}, \mathrm{Mn}$ - indeed appear to be underabundant by a factor of four to five. Calcium, and probably scandium, appear to be even more underabundant. On the other hand, several elements favored in the s-process, namely $\mathrm{Ce}$, $\mathrm{Sr}, \mathrm{Zr}, \mathrm{Sm}$, appear to be slightly to moderately overabundant.

\section{TABLE I}

Element abundances in the secondary of $U$ Sagittae (listed in order of decreasing accuracy) (Naftilan, 1975)

\begin{tabular}{lllll}
\hline Element & $\begin{array}{l}\text { Solar abundance } \\
\text { (from Allen, 1974) }\end{array}$ & $\begin{array}{l}\text { C.O.G. } \\
\text { results }\end{array}$ & $\begin{array}{l}\text { Synthetic } \\
\text { spectra } \\
\text { results }\end{array}$ & $\Delta \log N$ \\
\hline $\mathrm{Fe}$ & 7.60 & 7.0 & 6.8 & -0.7 \\
$\mathrm{Ti}$ & 5.13 & 4.7 & 4.4 & -0.6 \\
$\mathrm{Cr}$ & 5.85 & 5.4 & 5.2 & -0.55 \\
$\mathrm{Mn}$ & 5.40 & 5.0 & 4.5 & -0.7 \\
$\mathrm{Sm}$ & 1.48 & - & 2.0 & +0.5 \\
$\mathrm{La}$ & 1.6 & - & 1.7 & +0.1 \\
$\mathrm{Ca}$ & 6.30 & - & 5.0 & -1.3 \\
$\mathrm{Mg}$ & 7.42 & 4.9 & 6.4 & $?$ \\
$\mathrm{Co}$ & 5.1 & - & - & -0.7 \\
$\mathrm{Sr}$ & 2.85 & - & 3.0 & +0.15 \\
$\mathrm{Ce}$ & 1.1 & - & 2.0 & +0.9 \\
$\mathrm{Y}$ & 1.8 & - & 1.15 & -0.65 \\
$\mathrm{Sc}$ & 3.22 & - & 2.4 & -0.8 \\
$\mathrm{Zr}$ & 2.5 & & 2.9 & +0.4 \\
\hline
\end{tabular}


If these results are warranted by the more detailed study presently being conducted by Naftilan and Plavec, important implications will follow. There is little doubt that the subgiant in USge once was the more massive component and has been stripped of its outer layers. What we observe now is the deep interior of the original star - how far deep we are looking will require a detailed study of the system - and it appears that this interior deviates in its chemical composition from the solar surface composition.

Now everybody is aware of the current painful problem with the apparent lack of solar neutrinos. The standard solar model postulates a flux of $5.6 \pm 1.8 \mathrm{SNU}$, while the observed value does not surpass 1 SNU. In a broad discussion of the discrepancy, Ulrich (1974) considers also a model - originally devised by Bahcall and Ulrich (1971) - in which the abundance of metals in the solar interior is lower than in the solar atmosphere. He found that an underabundance by a factor of 10 in the interior would give $1.4 \pm 0.35$ SNU, much closer to the observed neutrino flux. Now the analysis of U Sge does point in the correct direction. The underabundances are probably less than required by the Bahcall-Ulrich model of the Sun. Moreover, this model also requires an uncomfortably small initial value for helium, $Y=0.10$ instead of the generally assumed value $Y=0.26$. I do not claim that here is a partial solution to the solar neutrino problem. I only want to stress that the abundance analysis of the Algols is clearly very important.

It is our intention to pursue this problem further. How much can the red spectra help? In stars like U Sge, U Cep, or AW Peg, blending is no longer serious at Ha. However, there are only few and relatively weak lines of Fe I and $\mathrm{Ca} I$ there. This region can be used only as a check on results derived from spectral regions of shorter wavelengths. On the other hand, shortwards of about $\mathrm{H} \beta$, spectral lines of the secondary begin to become seriously blended. A preliminary study indicates that the wavelength interval between $\mathrm{H} \beta$ and the $\mathrm{D}$ lines promises to be the best compromise.

We are well aware that a very good spectrum is a necessary but not always a sufficient condition for successful abundance analysis. The binary nature invokes a number of specific conditions. Although the primary component is completely eclipsed during the total phase, the spectrum of the secondary may still be contaminated by the light of the circumstellar disk which so very often surrounds the primary star, or by scattering of the light of the primary in the circumstellar envelope of the secondary. Recent discussion of the emission lines in UCep (Crawford and Plavec, 1975) indicates that this danger may be quite real in U Cep - but USge is probably less affected. Moreover, the calculation of the model atmosphere to fit the spectrum may be difficult as we have to take into account possible strong mass flux through the atmosphere, possible turbulence, sometimes fairly fast rotation, and the specific variable boundary conditions arising from the potential field of the binary system.

Of interest are the spectral types of the secondary components. We have so far observed nine stars, of which six have not been observed before. Table II lists the observed secondary component, region where its spectrum was studied, assigned spectral type, and comments. Typically these secondary stars have relatively broad lines for their spectral type, indicating that they are rotating in synchronism with orbital motion, or possibly even faster (RX Gem). The best lines are those of AW Peg B; this is probably an effect of low-gravity which makes the star a luminous giant, because its mass is quite small. This system is similar to AS Eri.

We believe that we have succeeded in solving the puzzle of the spectral type of the 
TABLE II

Classification of some Algol secondaries

\begin{tabular}{lllll}
\hline Star & $\begin{array}{l}\text { Spectral } \\
\text { region }\end{array}$ & $\begin{array}{l}\text { Spectral } \\
\text { type }\end{array}$ & $\begin{array}{l}\text { Uncertainty } \\
\text { in subclasses }\end{array}$ & Comment \\
\hline U Cep & H $\alpha$ & G 8 III-IV & \pm 1 & broad lines \\
RY Per & Ca II IR & F 0 III & \pm 2 & sharp lines \\
T Tau & Ca II IR & A 1 III-IV & \pm 1 & broad lines \\
RX Gem & Ca II IR & K 1: & $+3,-4$ & broad lines \\
TX UMa & Ca II IR & G 0 III-IV & \pm 1 & broad lines \\
U CrB & Ca II IR & G 0 III-IV & \pm 2 & broad lines \\
U Sge & H $\alpha$ & G 2 III-IV & \pm 2 & broad lines \\
S Equ & H $\alpha$ & F 9 III-IV & \pm 1 & sharp lines \\
AW Peg & H $\alpha$, Ca II IR & F 2: e pec & - & sharp, strong \\
\hline
\end{tabular}

primary component in V 367 Cyg. The system has been much observed and studied, but it seems to us that the Ca II infrared triplet gives a more definite picture of the spectrum of the primary star, and that it is a B8 Ia supergiant, very similar to Rigel.

\section{Circumstellar Material}

In order to get higher quality data on the interacting binaries, we have begun a systematic survey of the $\mathrm{H} \alpha$ line in Algols, with the Lick equipment described above. To date, we have observed some 40 semi-detached systems. Among them, $60 \%$ show some emission at least at a certain phase. In $40 \%$ of the systems, the emission appears to be permanent and is visible also outside of eclipse either as a genuine emission line or as a clear distortion of the complex $\mathrm{H} \alpha$ profile. Table III is a preliminary list of the observed Algols.

Some general conclusions can be drawn from this preliminary material. Systems of longer period tend to have stronger and more permanent emission. No doubt this is mainly due to the larger size of the Roche lobe around the primary, mass-accreting component. Another reason is, however, that the size of the accreting component is smaller compared to the dimensions of the system, and the flowing gas tends to form a disk around it rather than to impact on it. It appears that the attempt at a quantitative formulation of the condition for disk formation as expressed by Kł 1 (1971) is promising: U Cep was observed to display very prominent $\mathrm{H} \alpha$ emission in 1974 (Plavec and Polidan, 1975; Batten et al., 1975), while equally good observations of U Sge failed to show more than a marginal trace of emission. However, a big unknown here is the variability of the phenomenon, which is strongly suspected in U Cep and may be the rule rather than exception, in particular in the shorter-period systems.

We have so far observed four different types of circumstellar envelopes:

(1) The systems W Ser, V 367 Cyg, and BM Cas - the two former long known for a large quantity of circumstellar gas - have huge clouds surrounding the whole system. $\beta \mathrm{Lyr}$, according to the ultraviolet spectra obtained by Copernicus (Hack et al., 1975), may be another such system.

(2) A typical Algol system appears to have less gas. In a crude approximation, its distribution may then be described by Joy's model of a ring surrounding the primary star (Joy, 1942). That Joy's model interprets the observations qualitatively very well, is nicely seen from the sequence of spectra of UCep taken by us in August 1974, and 
TABLE III

Algols at $\mathrm{H} \alpha$

$(\mathrm{A}=$ absorption, $\mathrm{E}=$ emission $)$

\begin{tabular}{|c|c|c|c|c|}
\hline Star & $\begin{array}{l}\mathrm{H} \alpha \text { at } \\
\text { max. light }\end{array}$ & $\begin{array}{l}\text { H } \alpha \text { at } \\
\text { prim. ecl. }\end{array}$ & Type & $\begin{array}{l}\text { Comment on } \\
\text { variability }\end{array}$ \\
\hline SX Cas & $\mathbf{E}$ & - & disk? & profile varies \\
\hline BM Cas & E & - & cloud? & - \\
\hline U Cep & $A$ or $E$ & $\mathbf{E}$ & disk & profile, strength \\
\hline DM Per & $\mathbf{A}$ & $\mathbf{A}$ & - & no change \\
\hline RY Per & $\mathbf{E}$ & $\mathbf{E}$ & disk & profile, strength \\
\hline RZ Cas & $\mathbf{A}$ & - & - & no change \\
\hline RS Ari & $\mathbf{A}$ & - & - & no change \\
\hline$\beta$ Per & A & - & - & slight changes \\
\hline HR 1056 & A shell & - & disk? & no change \\
\hline$\lambda$ Tau & A & - & - & - \\
\hline RW Tau & A & E & disk & profile? \\
\hline HU Tau & A & - & - & - \\
\hline RZ Eri & A & - & - & - \\
\hline RX Gem & $\mathbf{E}$ & E & disk & profile, strength \\
\hline AU Mon & $\mathbf{E}$ & - & disk & profile \\
\hline RY Gem & $\mathbf{E}$ & - & disk & - \\
\hline UX Mon & E & - & disk & - \\
\hline TX UMa & E & $\mathbf{A}$ & shock & profile, strength \\
\hline TT Hya & $\overline{\mathbf{E}}$ & $\mathbf{E}$ & disk & profile, strength \\
\hline SX Hya & A & - & - & profile? \\
\hline$\delta \mathrm{Lib}$ & A & $\mathbf{A}$ & - & no changes \\
\hline $\mathrm{U}$ CrB & E & $\mathbf{E}$ & disk & profile, strength \\
\hline UZ Lib & E & $\overline{-}$ & disk? & profile? \\
\hline TW Dra & $\mathbf{A}$ & $\mathbf{E}$ & - & - \\
\hline AI Dra & A & - & - & - \\
\hline u Her & A & - & - & - \\
\hline V 393 Sco & A? & - & - & - \\
\hline V $453 \mathrm{Sco}$ & $\mathbf{E}$ & $\overline{-}$ & disk? & profile? \\
\hline W Ser & $\mathbf{E}$ & E & cloud & strength \\
\hline$\mu \mathrm{Sgr}$ & E & $=$ & - & profile \\
\hline RZ Sct & $\mathrm{E}$ & E & disk & strength \\
\hline RY Sct & E & - & cloud? & - \\
\hline EG Ser & $\mathbf{A}$ & - & - & - \\
\hline V $356 \mathrm{Sgr}$ & $\mathbf{A}$ & $=$ & - & - \\
\hline$\beta$ Lyr & $\mathbf{E}$ & E & cloud? & no changes \\
\hline V $342 \mathrm{Aql}$ & $\mathrm{E}$ & - & - & - \\
\hline RS Vul & A & - & - & - \\
\hline U Sge & $\mathbf{A}$ & A & - & no change? \\
\hline SW Cyg & $\mathbf{A}$ & - & - & - \\
\hline VW Cyg & $\mathbf{E}$ & - & - & - \\
\hline W Del & $\mathbf{E}$ & $\overline{-}$ & shock? & strength \\
\hline S Equ & $\mathbf{A}$ & $\mathbf{E}$ & disk & profile, strength \\
\hline V 367 Cyg & $\mathbf{E}$ & E & cloud & strength \\
\hline AW Peg & $\mathbf{E}$ & E & chromosphere? & strength \\
\hline AR Lac & A & - & - & - \\
\hline AN And & $\mathbf{A}$ & - & - & - \\
\hline
\end{tabular}

shown in Figure 1 (Plavec and Polidan, 1975). However, preliminary analysis of these spectra presently conducted by R. Crawford (Crawford and Plavec, 1975) indicates that simple model of a flat disk within the Roche critical lobe cannot completely explain the observed emission line profile and its changes with phase. 


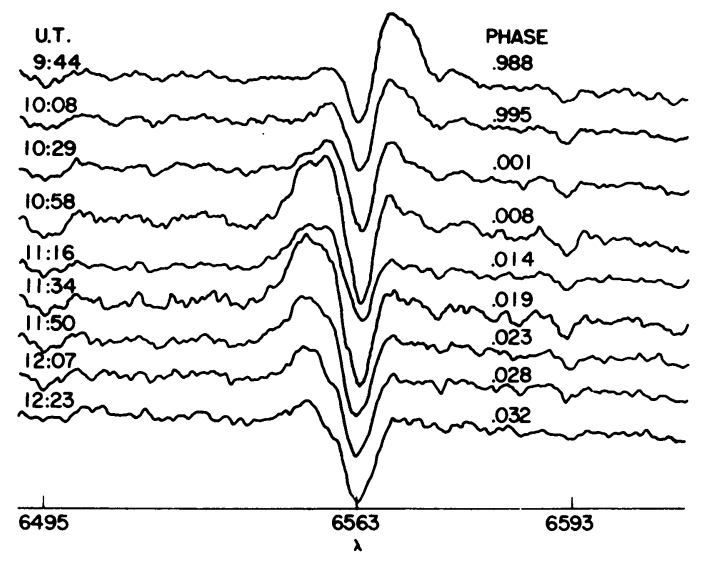

Fig. 1. Density tracings of nine spectra of U Cephei taken at the time of primary eclipse on 1974, August 8 . Tracings are superimposed so that the absorption cores nearly coincide. Laboratory wavelengths in angstroms are indicated on the $x$-axis. Phases are in fractions of the period. Note the strong red emission lobe at $\mathrm{H} \alpha$ prior to the mid-eclipse, and the strong violet emission lobe immediately after mid-eclipse.

(3) The third type of circumstellar phenomena has so far been observed only in TX UMa. Here the emission line appears only at certain very definite phase intervals outside of eclipse, and is much sharper than in the case of the disk. We suspect we are observing here the radiation from an accretion shock postulated by Lubow and Shu (1975).

(4) Yet another type of phenomenon is probably displayed by AW Peg. Here the emission is seen at all phases, but it appears to be associated with the cooler, mass-losing component. The emitting region may have the character of an extended chromosphere.

Although we have already accumulated over 1100 spectrograms in the red and near infrared regions, and observed more than 200 objects altogether, we are still very far from having a clear picture of the phenomena.

\section{From the Algols to the Be Stars}

In a typical short-period Algol system such as U Cep or RW Tau, the emission at $\mathrm{H} \alpha$ is observable only during the deep phase of the primary eclipse. Then in U Cep it exceeds about twice the continuum level of the G star. This means that the light of the circumstellar disk is only a fraction of the light of the primary component at the corresponding wavelengths. If the system were not totally eclipsing, we would know nothing about the circumstellar material. However, as has already been said, if one goes to systems of longer period, the emission becomes much stronger and is visible in full light. Such a case is, for example, TT Hya, with a period of seven days. Here the primary component has been classified as A3 III e, and the emission line at $\mathrm{H} \alpha$ persist through all phases. Moreover, our observations show that the emission line profile undergoes a kind of periodic variation with orbital period - a variation which is reminiscent of the classical $V / R$ variation in Be stars. The dimensions and masses in TT Hya are not yet fully known, but they have 
been estimated (Hall, 1974) and the radius of the critical Roche lobe around the primary may be about 12 solar radii.

What size of the emitting disk will give a full-fledged Be phenomenon? There is no consensus of opinion on the size of Bestar envelopes, but the recent trend has been towards a larger extent of the emitting regions. If the envelope is also to imprint deep sharp absorption lines as in shell stars, the estimate is that the deep cores are affected even by material some 100 stellar radii above the photosphere of the shell star 1 Del (Marlborough and Cowley, 1974); since the star is about B8, this would imply an envelope of about $300 R_{\odot}$ thickness. For an ordinary. Be star without the shell absorption lines, the estimate may be some $50 R_{\odot}$ (Marlborough, 1969). A typical Be star is of spectral type about B3. If we assume its mass to be about $8 M_{\odot}$, and take the mass ratio in the system to be $7: 1$, the required distance between the two stars will turn out to be some $100 R_{\odot}$, and the corresponding period about $\mathbf{4 0}$ days. Even if the secondary component in such a hypothetical binary system fills its critical Roche lobe and is therefore a giant with a radius of about $20 R_{\odot}$, the geometrical probability that the system will be observed as an eclipsing binary is not large, mainly because the primary component has a very small relative radius $\left(R_{1} / A \cong 0.06\right)$. Nevertheless, the probability of eclipses is not negligible. In the case we have been studying, total eclipses should occur for all inclinations greater than $81^{\circ}$, and this includes $15 \%$ of all the systems provided their orbital planes are oriented at random. This is in sharp disagreement with observations; no bright bona fide $\mathrm{Be}$ star is known to undergo deep eclipses.

Therefore we conclude that mass transfer in Algols of longer periods may probably produce a bona fide Be star, but it does not seem possible to account in this way for the whole population of Bestars. In other words, we are reiterating again the suggestion made several times in the past by one of us (see, e.g., Plavec and Horn, 1969; Plavec, 1970), that duplicity and mass transfer may play an important role in the Be star phenomenon, but we find it less easy to accept the idea proposed by Kł $\mathrm{iz}$ and Harmanec (1975) that all Be stars are interacting binaries.

It appears that the infrared $\mathrm{Ca}$ II triplet in emission may be an important indicator of an interacting binary (Polidan, 1975). If so, then among the well-known Be stars and shell stars only some would appear to be interacting binaries (e.g. $\phi$ Per, $\psi$ Per, HR 2142 , Pleione, $48 \mathrm{Per}, v \mathrm{Cyg}, \pi \mathrm{Aqr}, 66 \mathrm{Oph}$ ), while the absence of this emission in other objects (such as $\gamma$ Cas, $\zeta$ Tau, $48 \mathrm{Lib}, \chi$ Oph, 1 Del, 59 Cyg, $\epsilon$ Cap, 31 Peg, EW Lac, $\beta$ Psc) might indicate a different origin.

Of the total sample of over 100 Be stars surveyed by us, less than $20 \%$ display the calcium triplet emission. The lines are optically thick, which implies that emission at $\mathrm{H}$ and $\mathrm{K}$ should be even stronger, since these lines have the same upper level. No emission at $\mathrm{H}$ or $\mathrm{K}$ is observed, however. It is therefore difficult to develop a physically realistic model which does not invoke the presence of a cool companion to the Be star (Polidan, 1975). Here we find a strong argument for duplicity, and observational selection may be responsible for seeing the triplet emission in only $20 \%$ of cases. Again, it seems hardly possible to go very much above this $20 \%$ fraction.

Thus, in essence, we are proposing a scheme in which the Be stars would have double origin: some may be single objects, others may be the mass-accreting components of interacting binaries. In fact, there may be a third group comprising Herbig's young Ae objects where the emitting envelope is the remnant of the original cocoon in which the 
star was embedded at the time of its birth. This scheme of diversity may not be so unsatisfactory as one might think at first sight. After all, we only postulate the presence of an extended circumstellar disk around an early-type star, and such a disk could conceivably originate in different ways. The single-star hypothesis has its difficulty in explaining the mechanism of ring formation and its dynamical support. It will be interesting to see if the stellar wind may be the mechanism aiding rapid rotation. The binary star hypothesis explains - at least qualitatively - the presence of the disk by the influx of material with excess angular momentum, and the rapid rotation of the star by partial oblique impact of the same material (Plavec, 1970; van de Heuvel, 1970). However, any more detailed models are missing.

We believe that the binary Be stars are close relatives of the Algols, with longer periods (typically one or several months), and with fairly large mass ratios. We would like to call attention to Figure 2, where the distribution with spectral type is shown for two kinds of eclipsing binaries: the detached systems with both components on the main sequence (briefly the MS binaries), and the semidetached systems (Algols). The binaries have been grouped according to the spectral type of the primary (i.e. hotter and more massive)

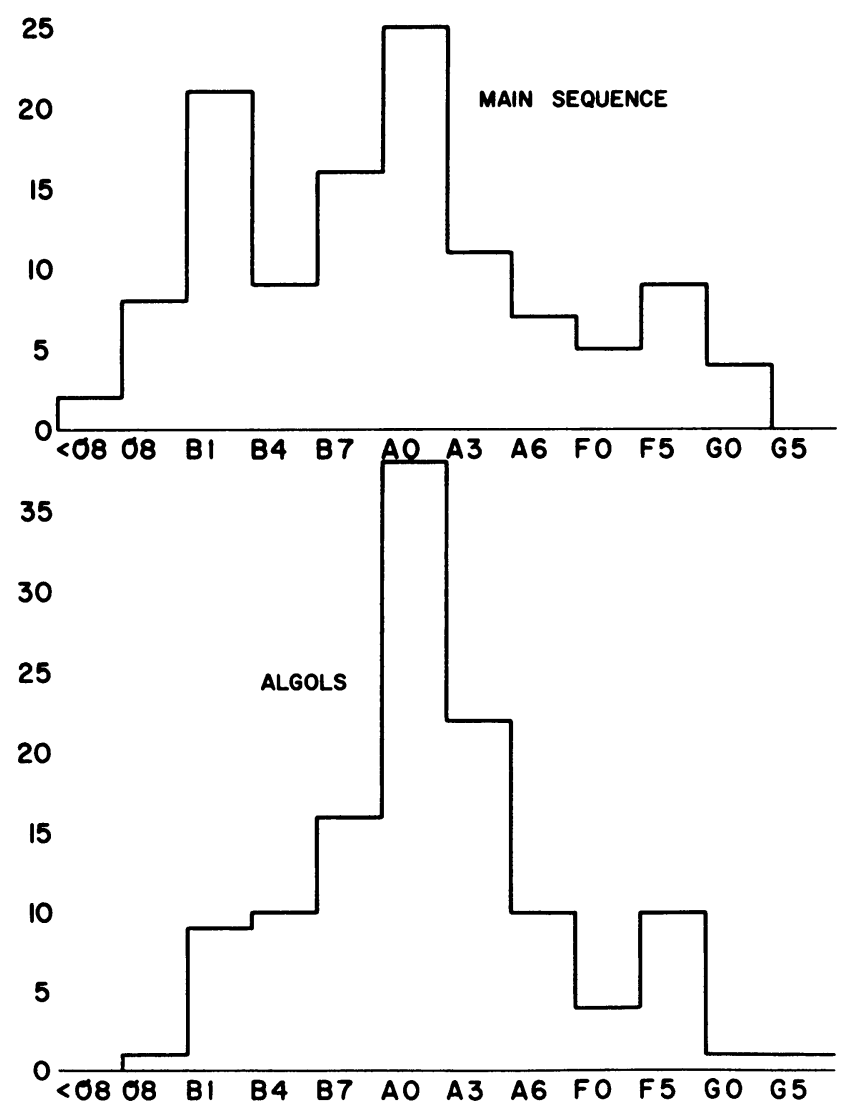

Fig. 2. Distribution of primary components with spectral type for two kinds of eclipsing binaries. 
component. The distribution is affected by the well-known inhomogeneity of the MK classification; for example, spectral types of B4-B6 are underrepresented even among single stars in the Bright Star Catalogue and in the HD catalog, according to a survey made kindly for us by Dr J. Mead. It is better to compare the relative numbers of the two kinds of eclipsing binaries. Two maxima of occurrence coincide: a very pronounced one at $\mathbf{A} 0-\mathbf{A} 2$, and a secondary peak at F5-F9. This coincidence may mean that the process of mass transfer almost completely reverses the masses, as is indicated by model calculations.

If, then, the MS systems are progenitors of the Algols of about the same spectral type, why don't we observe enough Algols at B1-B3, where there is another pronounced peak in the number of the MS systems? One is tempted to suspect that here the mass transfer process may lead to Be stars which tend to occur most frequently for these spectral sub. classes. However, we have as yet no model calculations that would support this conjecture. There is one big unknown which prevents us from predicting how numerous are the binary Be stars, and I am sad to say that even this Symposium has not removed the impasse.

We are talking about the stability of the accretion disks. If the accretion disks are governed primarily by high viscosity, they will dissipate almost instantaneously as soon as the mass transfer ends. In this case a binary Be star must be a system where the mass transfer is still going on. Our chances of observing such a system are low because of the short timescale of the transfer. Moreover, the argument about the probability of eclipses used above comes here in full strength. Thus, in this case, both theoretical and observational arguments would indicate that binary Be stars should be rather rare.

If, however, the model of accretion disks presented and defended here by Shu and by Flannery is more correct, then the disk would persist for a relatively long time even after the gas supply has been turned off. In this case one can have a Be star in a binary which interacted in the past but now the mass-losing component is contracting to become or has already become a white dwarf or a helium star. This makes the timescale considerably longer. Moreover, there would be practically no eclipses to expect because the companion is too small. In this case, the population of interacting binaries among the Be stars can be quite large.

It appears that the problem of the accretion disks is crucial, and should be attacked both theoretically and observationally. Let us mention briefly that if the stellar wind plays an important role here, the picture may be changed again. In the binary model of Be stars, we do not need red giants filling the critical Roche lobe, for they can lose mass through the stellar wind - provided the mass loss is sufficient to produce a gaseous ring around the other component. On the other hand, stellar wind may make it considerably easier to explain the Be star phenomenon by a single-star model, and make the binary model less relevant.

\section{Very Deep Stellar Eclipses}

The idea that at least some Be stars or shell stars are interacting binaries would be strongly corroborated if we could find more binary objects among them. In the case of the two safely established cases, AXMon and 17 Lep, the opponents of the binary hypothesis can object that the two systems are too peculiar to be representative of shell 
stars. We have been undertaking an extensive search for cool components by studying the near infrared spectra; we will present partial results in the near future.

Here we would like to propose another test of the hypothesis, namely a search for long-period eclipsing binaries with deep eclipses. The Algols of shorter period appear to have certain restrictions on the spectral differences between the two components, as shown very well in the recent statistical survey by Koch (1973). Our list of 123 Algols contains 20 systems with primaries earlier than B7. Most of them have components of spectral type A (like $\lambda$ Tau, V $356 \mathrm{Sgr}$, and Z Vul). For these, the eclipses are only moderately deep. The coolest companions observed are in RS Vul (about G5) and U CrB (about G0); these systems would have very deep eclipses if they were not partial. Because of this partiality, the deepest eclipses among the known systems are those of U Cep (B6: + G8) and RW Tau (B8 + K0:).

This restriction on the spectral type of the mass-losing star is compatible with model calculations. However, for the wider systems like AX Mon and 17 Lep, such a limit is probably absent. The B9 primary in 17 Lep is combined with an M1 giant. Using the model adopted by Cowley (1967), one finds that if we observed the system edge-on $\left(i=90^{\circ}\right)$, the eclipses would be more than 2 mag deep in $B$, and would last for about 50 days out of the period of 260 days. Similarly, central eclipses would again be more than two magnitudes deep for AX Monocerotis, which combines a B3: star with a K0: giant.

However, these eclipses are in fact exceptionally shallow for systems with such different spectral types of the components. The reason is the selection effect that enables us to recognize them as binaries: the cool secondary is a large giant, so that it gives enough light for its spectral lines to be visible in the region around $\mathrm{H} \alpha$. Other binaries, like XX Oph, show greater disparity in the $V$ or $R$ magnitudes, and traces of the spectrum of the secondary are not visible until farther off in the infrared. Thus we can anticipate the existence of long-period systems where the eclipses could be substantially deeper than two magnitudes.

It is almost certain that some of these deeply eclipsing, long period binaries must exist at least among the fainter variables. It is tempting to speculate what kind of variability would be reported if such an object were observed only occasionally on patrol plates. It is not impossible that it might be at first classified as an early-type variable of the $\mathrm{R} \mathrm{CrB}$ class.

\section{Acknowledgements}

In many of the Lick observations reported here, Dr Geraldine J. Peters took an important part. In preparing this paper, we profited from many discussions with Drs Popper, Ulrich, Peters, and Naftilan. This work has been supported by NSF grant MPS 74-04194 A 01 . We thank Marietta Eaker for typing the manuscript, and Kathy Sedwick for drafting the Figures.

\section{References}

Bahcall, J. N. and Ulrich, R. K.: 1971, Astrophys. J. 170, 593.

Baldwin, B. W.: 1973, Publ. Astron. Soc. Pacific 85, 714.

Batten, A. H., Fisher, W. A., Baldwin, B. W., and Scarfe, C. D.: 1974, Nature 253, 174. 
Bodenheimer, P. and Ostriker, J. P.: 1970, Astrophys. J. 161, 1101.

Bond, H. E.: 1972, Publ. Astron. Soc. Pacific 84, 839.

Cowley, A. P.: 1967, Astrophys. J. 148, 667.

Crawford, R. C. and Plavec, M.: 1975, Bull. Amer. Astron. Soc. 7, 462.

Doremus, C.: 1970, Publ. Astron. Soc. Pacific 82, 745.

Gaposchkin, S.: 1946, Harvard Bull., 918.

Hack, M., Hutchings, J. B., Kondo, Y., McCluskey, G. E., Plavec, M., and Polidan, R. S.: 1975, Astrophys. J. 198, 453.

Hall, D. S.: 1967, Astron. J. 72, 301.

Hall, D. S.: 1971, Bamberg Variable Star Colloq., p. 217.

Hall, D. S.: 1974, Acta Astron. 24, 215.

Joy, A. H.: 1930, Astrophys. J. 71, 336.

Joy, A. H.: 1942, Publ. Astron. Soc. Pacific 54, 35.

Koch, R. H.: 1960, Astron. J. 65, 139.

Koch, R. H.: 1972, Publ. Astron. Soc. Pacific 84, 5.

Koch, R. H.: 1973, Astron. J. 78, 410.

Kopal, Z.: 1959, Close Binary Systems, Chapman and Hall, chap. VII.

Kł́̂̉, S.: 1971, Bull. Astron. Czech. 22, 108.

Kłî̀, S. and Harmanec, P.: 1975, Bull. Astron. Czech. 26, 65.

Lubow, S. H. and Shu, F. H.: 1975, Astrophys. J. 198, 383.

Marlborough, J. M.: 1969, Astrophys. J. 156, 135.

Marlborough, J. M. and Cowley, A. P.: 1974, Astrophys. J. 187, 99.

McNamara, D. H.: 1967, Astrophys. J. 149, 723.

Miner, E. D.: 1966, Astrophys. J. 144, 1101.

Naftilan, S. A.: 1975a, Publ. Astron. Soc. Pacific 87, 321.

Naftilan, S. A.: 1975b, Bull. Amer. Astron. Soc. 7, 476.

Plavec, M.: 1970, in A. Slettebak (ed.), Stellar Rotation, D. Reidel Publ. Co., Dordrecht, Holland, p. 133.

Plavec, M.: 1973, IAU Symp. 51, 216.

Plavec, M. and Horn, J.: 1969, in M. Hack (ed.), Mass Loss from Stars, D. Reidel Publ. Co., Dordrecht, Holland, p. 242.

Plavec, M. and Polidan, R. S.: 1974, Nature 253, 173.

Polidan, R. S.: 1975, Bull. Amer. Astron. Soc. 7, 451.

Popper, D. M.: 1973, Astrophys. J. 185, 265.

Popper, D. M. and Plavec, M.: 1974, Bull. Amer. Astron. Soc. 6, 334.

Popper, D. M. and Plavec, M.: 1976, Astrophys. J. 205, 462.

Refsdal, S., Roth, M. L., and Weigert, A.: 1974, Astron. Astrophys. 36, 113.

Struve, O.: 1950, Stellar Evolution, Princeton Univ. Press, p. 27.

Ulrich, R. K.: 1974, in C. Baltay (ed.), Neutrinos 1974, Amer. Inst. of Physics, p. 259.

van de Heuvel, E. P. J.: 1970, in A. Slettebak (ed.), Stellar Rotation, D. Reidel Publ. Co., Dordrecht, Holland, p. 178.

Wilson, R. E.: 1972, Astrophys. Space Sci. 19, 165.

\section{DISCUSSION}

Wilson: Dr Hall deserves a bow, because the object you now find in BM Ori is precisely what he said would be found about five years ago.

Plavec: Yes, indeed, this is properly acknowledged in the article submitted to the Astrophysical Journal.

Smak: First a comment on the underabundancies obtained via the narrow band photometry. It appears that, since the so-called subgiants are actually, in terms of the surface gravity, supergiants, many of these results were either wrong, because they resulted from using the single subgiants as standards, or at least uncertain, due to the lack of suitable supergiants as standards. Now a question: how large is the danger of emission lines from the circumstellar material contaminating the lines of the secondary in U Sge and leading to the underabundance you just reported?

Plavec: I do not think the danger is large in U Sge since there are virtually no emissions. Nevertheless, we are aware of this danger, which may be quite serious in other systems with well-developed disks. 
Geyer: Your talk about possible deep eclipses reminds me of a system I came across in Cepheus some $15 \mathrm{yr}$ ago. It is an $11 \mathrm{mag}$ star which drops in minimum beyond $17 \mathrm{mag}$.

Shaviv: I want to refer to the comment on the solar neutrino value obtained with models of different $Z$. You can assume either that the sun is an unusual star with a discontinuity in $Z$ in the interior or that all main-sequence stars have it for some reason (like accretion). If you try to fit the main-sequence of some galactic clusters with model that have a discontinuity in $Z$ you find that there exists an upper limit to the allowed discontinuity in order to preserve agreement with observations. This upper limit on the discontinuity is not sufficient to reduce the neutrino flux to below 1 SNU. You can assume that $\mathrm{p}+\mathrm{p}$ has a resonance at an energy of $2 m_{p} c^{2}$ which contributes a small correction (say a factor of $2-3$ ) to the reaction rate at thermal energies. This may be supported by some recent results on ${ }^{3} \mathrm{He}+{ }^{3} \mathrm{He} \rightarrow \mathrm{He}^{4}+2 \mathrm{p}$ scattering. 